\title{
Can We Use Syndromic Surveillance Data to Identify Primary Care Visits to NYC EDs?
}

\author{
Jessica Athens* \\ New York City Department of Health and Mental Hygiene, New York City, NY, USA
}

\section{Objective}

To develop a syndrome classification based on patient chief complaint to (1) estimate the proportion of primary care-related emergency department (ED) visits in New York City (NYC) hospitals and (2) explore predictors of such visits.

\section{Introduction}

NYC EDs saw nearly 4 million visits in 2011. Studies have demonstrated that non-urgent visits can account for more than $50 \%$ of visits to EDs [1,2]. Designed to provide rapid diagnosis and first-line treatment of serious illness, EDs often function as a primary care site due to their accessibility. Unfortunately, use of EDs for primary care may affect their ability to meet the needs of severely ill patients.

\section{Methods}

We examined syndromic surveillance data from 45 hospitals in NYC for 2011 and classified visits into a primary care syndrome based on the chief complaint field. Data from 4 hospitals were omitted due to data quality issues, as were records from non-NYC residents. Primary care (PC) syndrome visits included visits recorded as referrals, screenings, suture removal/dressing changes, or medication refills; records with a blank or non-informative (e.g. "X") chief complaint field were omitted from analysis. Using unique patient IDs, we identified patients who visited the same ED multiple times in the previous 12 months. A hierarchical generalized linear mixed effects model with hospital-level random effects was used to explore patient characteristics associated with PC syndrome visits. The model included a random intercept for hospital and the following covariates: duplicate visit, patient gender and age group (ages 0-4, 5-17, 18-64, and $65+$ ), and time of visit (midnight to $8 \mathrm{AM}, 8 \mathrm{AM}$ to midnight). Covariates for month and day of week were included to control for temporal trends in ED visits. Model parameters were estimated by maximum likelihood. Estimation was performed in SAS version 9.2 [3] using the GLIMMIX procedure.

\section{Results}

Citywide, $7.5 \%(\mathrm{~N}=190,431)$ of visits to EDs during 2011 were classified as PC syndrome visits, but varied by hospital with a median of $4.6 \%$ (IQR: $3 \%$ to $9 \%$ ) across hospitals. The average proportion of PC syndrome visits varied by hospital. Of the 45 hospitals included in the analysis, 18 had a lower baseline, 13 were the same, and 14 had a higher baseline than the city mean. Hospitals with a larger census had a larger proportion of PC syndrome visits.

Age had a significant effect on the odds of a PC syndrome visit; ages $0-4$ had the greatest odds of a PC syndrome visit relative to the $65+$ age group. Visits from patients ages 5-17 and 18-64 were also more likely to be primary care visits. Patients with repeat visits were more likely to have PC syndrome visits. Female gender and early morning visits $(12 \mathrm{~A}-8 \mathrm{~A})$ were associated with lower odds of a PC syndrome visit.

\section{Conclusions}

With limited detail on patient visits, our syndrome likely undercounts primary care visits to EDs. However, the relationships between our explanatory variables - age, time of day, and duplicate visitsand $\mathrm{PC}$ syndrome visits are consistent with the literature on ED usage for primary care. Gender is an exception [1], but earlier findings may be confounded by the fact that females seek health care more frequently in general. The variation in PC syndrome visits among NYC EDs is significant and may be explained by hospital or community measures not captured in our model, such as clinic wait times, ED capacity, or insurance coverage. In fact, disparities in such predictors of PC syndrome visits could be targets for interventions. Our ability to replicate previous findings on the use of EDs for primary care visits suggests that syndromic data may be a near real-time data source for following trends in such visits.

Predictors of PC Syndrome Visits

\begin{tabular}{|c|c|}
\hline Covariate & OR (95\% CI $)$ \\
\hline Duplicate visit & $1.84(1.82-1.86)$ \\
\hline Female & $0.84(0.83-0.85)$ \\
\hline Age 0-4 & $2.15(2.11-2.19)$ \\
\hline Age 5-17 & $1.55(1.52-1.58)$ \\
\hline Age 18-64 & $1.26(1.24-1.28)$ \\
\hline Age 65+ & Reference group for age \\
\hline Early AM (12A-8A) & $0.85(0.84-0.86)$ \\
\hline
\end{tabular}

All covariates significant at $\mathrm{p}<.001$.

\section{Keywords}

Misuse of emergency medical services; Primary care; Variation in emergency department use

\section{References}

1. Carret M, Gastal Fass A, Rodrigues Domingues A. Inappropriate use of emergency services: a systematic review of prevalence and associated factors. Cad. Saúde Pública. 2009;25(1): 7-28.

2. Tang N, Stein J, Hsia RY, Masselli JH, Gonzales R. Trends and characteristics of US emergency department visits, 1997-2007. JAMA. 2010;304(6):664-70.

3. SAS Institute, Inc., Cary, NC.

\section{*Jessica Athens}

E-mail: jathens@health.nyc.gov 